\title{
Efficient Isolation and Gene Expression Profiling of Small Numbers of Neural Crest Stem Cells and Developing Schwann Cells
}

\author{
Johanna Buchstaller, ${ }^{1}$ Lukas Sommer, ${ }^{1}$ Matthias Bodmer, ${ }^{1}$ Reinhard Hoffmann, ${ }^{2}$ Ueli Suter, ${ }^{1}$ and Ned Mantei ${ }^{1}$ \\ ${ }^{1}$ Institute of Cell Biology, Department of Biology, Swiss Federal Institute of Technology, CH-8093 Zürich, Switzerland, and ${ }^{2}$ Max-von-Pettenkofer-Institut, \\ D-80336 Munich, Germany
}

\begin{abstract}
Schwann cells develop from multipotent neural crest stem cells and are important for neuronal survival, maintenance of axonal integrity, and myelination. We used transgenic mice expressing green fluorescent protein in a tissue-specific manner to isolate viable, pure populations of neural crest stem cells and developing Schwann cells, which are not readily accessible by microdissection. Starting with the minute amounts of RNA obtained, a two-round amplification procedure was used to achieve reproducible DNA array hybridizations. We validated our screening procedure by comparisons with the literature and by in situ hybridization. Stage-to-stage comparisons and hierarchical clustering for neural crest and five stages of Schwann cell development suggest a wealth of candidates for genes involved in stem cell regulation and in early Schwann cell development. The combination of methods applied in this study should be generally useful for isolating and profiling other stem cell and difficult to isolate cell populations.
\end{abstract}

Key words: development; gene; glia; neural tube neural crest; neural plate; Schwann cell; sensory neurons; green fluorescent protein; GFP; fluorescence-activated cell sorting; FACS; peripheral nervous system; PNS; microarray; expression analysis

\section{Introduction}

When performing gene expression profiling of cells freshly isolated from an organ or tissue, homogeneity of the cell population is a major issue. Often the cells of interest are mixed with other cell types in a given anatomical location, and gross dissection may lead to the concomitant isolation and profiling of contaminating tissue (Smith and Greenfield, 2003). Recently, animals expressing fluorescent proteins under the control of various tissue-specific promoters have been used to retrieve specific cell types from a complex mixture of cells by fluorescence-activated cell sorting (FACS). In particular, developing and mature oligodendrocytes have been isolated from brains and spinal cords of mice expressing green fluorescent protein (GFP) under the control of the proteolipid protein (Plp) promoter (Fuss et al., 2000; Spassky et al., 2001). Because the Plp promoter is also active in Schwann cell (SC) precursor cells of the peripheral nervous system (PNS) (Griffiths et al., 1998), mice expressing GFP under its control appeared promising for exploring gene expression in early development of the PNS.

At approximately embryonic day (E) 9 in the mouse, neural

Received Sept. 4, 2003; revised Dec. 30, 2003; accepted Jan. 8, 2004.

This work was supported by the Swiss National Science Foundation and the National Center for Competence in Research "Neural Plasticity and Repair." We thank B. Zalc for transgenic mice, M. Schwab, M. Wegner, S. Mansour, A. Goffinet, and A. Gow for antibodies and riboprobes, and M. Marchionni for GGF2. We thankE. Niederer for assistance with the FACS, K. Mannigel for animal care, C. Lobsiger and R. Leimeroth for help with tissue dissections, and J. Relvas for critically reading this manuscript.

Correspondence should be addressed to Ned Mantei, Institute for Cell Biology, Swiss Federal Institute of Technology, Schafmattstrasse 18, CH-8093 Zürich, Switzerland. E-mail: mantei@cell.biol.ethz.ch.

DOI:10.1523/JNEUROSCI.4083-03.2004

Copyright $\odot 2004$ Society for Neuroscience $\quad$ 0270-6474/04/242357-09\$15.00/0 crest stem cells (NCSCs) detach from the dorsal neural tube and migrate along defined pathways to various tissues. These selfrenewing, multipotent cells give rise to neurons and glia of the PNS, but also to a variety of other differentiated cell types such as smooth muscle, cartilage, bone, and melanocytes (Le Douarin and Kalcheim, 1999). At E10, trunk NCSCs colonize the somites and aggregate to form dorsal root ganglia (DRG), in which progenitor cells differentiate first into sensory neurons and later into satellite glia. Neural crest-derived cells associate with sensory axons growing out from the DRG and with CNS motor axons, and differentiate into SC precursor cells, prominent at E12. These cells proliferate during their migration along the nerves, and by E16 have differentiated into immature or early SCs. Shortly before birth, SCs invade the axon bundles and associate in a oneto-one relationship with large-caliber axons. Shortly after birth, some of the SCs begin to form myelin sheaths, whereas others remain as nonmyelinating SCs (Anderson, 1997; Jessen and Mirsky, 1999, 2002; Le Douarin and Kalcheim, 1999; Sommer, 2001; Lobsiger et al., 2002).

Development of peripheral nerves depends on complex reciprocal interactions between SCs and neurons. Neuronally derived signals such as ligands of the Delta-Notch family (Morrison et al., 2000; Wakamatsu et al., 2000), neuregulins (Shah et al., 1994; Dong et al., 1995; Meyer and Birchmeier, 1995; Garratt et al., 2000; Leimeroth et al., 2002), transforming growth factor $\beta$ (TGF $\beta$ ) (Parkinson et al., 2001), and electrical activity (Stevens and Fields, 2000) induce fate determination of NCSCs and differentiation of developing SCs, and regulate their number in the peripheral nerve. In turn, survival of motor and sensory neurons 
during development depends on the presence of SCs, either through provision of trophic factors or via less direct effects (Riethmacher et al., 1997; Davies, 1998; Woldeyesus et al., 1999). SC-derived factors control the formation of the perineurial sheath, which protects and assures the integrity of axon bundles (Parmantier et al., 1999). Later in development, myelinating SCs organize the axonal architecture by regulating axonal caliber, by controlling the distribution of ion channels on the axonal membrane, and by influencing neurofilament phosphorylation in myelinated axons (Jessen and Mirsky, 1999; Scherer and Arroyo, 2002; Spiegel and Peles, 2002). After an injury, SCs are crucial for axonal regeneration (Terenghi, 1999), and in most demyelinating neuropathies the deleterious effects originate primarily from SCs (Suter and Scherer, 2003).

Early cell fate determination of NCSCs to SCs and the subsequent differentiation occur under the influence of multiple signals from the environment, which are integrated and together with intracellular factors are interpreted by the cells to elicit a cell type-specific response (Anderson, 2000; Morrison, 2001; Sommer, 2001). To learn more about the molecular mechanisms controlling these processes, and to identify novel components potentially involved in the dialogue between SCs and neurons, we undertook large-scale gene expression profiling of NCSCs and five stages of embryonic and perinatal SC development. Profiling of NCSCs should also improve understanding of stem cell biology in general, yielding candidate genes involved in regulation of self renewal, migration, and differentiation into a wide range of cell types.

Global expression profiling has been used to characterize the earliest delaminating stage of neural crest development in the chicken (Gammill and Bronner-Fraser, 2002) and late stages of myelination in the mouse (Nagarajan et al., 2002; Verheijen et al., 2003), but intermediate stages of PNS development have not yet been so characterized. Because acutely isolated NCSCs and developing SCs are not readily accessible without contaminating tissue ex vivo, we used FACS to purify viable cells from mice expressing GFP under the control of the Plp promoter in the developing PNS. The small amounts of RNA obtained from these cells were amplified by two rounds of in vitro transcription. Labeled complementary RNA (cRNA) was hybridized with Affymetrix GeneChips. To increase the predictive ability of our expression data we hybridized RNA from three to four separate batches of embryos or pups per developmental stage and performed extensive statistical analysis of the data set.

\section{Materials and Methods}

Transgenic mice. Plp-GFP transgenic animals (line 42) (Spassky et al., 2001) were provided by B. Zalc (Institut National de la Santé et de la Recherche Médicale U-495, Hôpital de la Salpêtrière, Paris). Heterozygous embryos or pups were obtained by mating homozygous transgenic males with C57BL/6 females, taking the date of the vaginal plug as E0.

Preparation of sections. Pregnant females were killed by exposure to $\mathrm{CO}_{2}$. Embryos were dissected, washed in PBS, and embedded in 3\% low-melting agarose. Sections $(200 \mu \mathrm{m})$ cut with a vibrating blade microtome were mounted and photographed immediately with a Zeiss Axioplan microscope and a digital camera.

Cell preparation and sorting. A fluorescent stereomicroscope (Leica, Nussloch, Germany) was used to dissect regions in which emigrating NCSCs were detected in the somites of E9.5 transgenic embryos. Tissue from two to three litters was incubated $12 \mathrm{~min}$ at $37^{\circ} \mathrm{C}$ in $1 \mathrm{ml}$ of HBSS with $0.05 \%$ trypsin (Invitrogen, San Diego, CA) and $3 \mathrm{mg} / \mathrm{ml}$ collagenase type III (Worthington Biochemical, Freehold, NJ). After quenching with $0.1 \mathrm{ml}$ of fetal calf serum (FCS), the material was triturated, centrifuged at $180 \times g$ for $5 \mathrm{~min}$, and resuspended in PBS-2\% FCS. Cell suspensions were prepared from sciatic nerves as in Jessen et al. (1994) and Lobsiger et al. (2001). Before sorting, the cell suspensions were passed through a 70 $\mu \mathrm{m}$ cell strainer (Falcon). For cell sorting we used a FACStar PLUS (Becton Dickinson, Heidelberg, Germany) and CellQuest software. Cells $\left(1-3 \times 10^{6} / \mathrm{ml}\right)$ were analyzed for forward scatter, side scatter, and GFP fluorescence with an argon laser (488 $\mathrm{nm}$ excitation, $520 \mathrm{~nm}$ emission). Dead cells and doublets were excluded by gating on forward and side scatter.

Cell culture. Culture of NCSCs at medium density (1000-2000/5 $\mu \mathrm{l})$ was performed as reported previously (Stemple and Anderson, 1992; Hagedorn et al., 1999). Embryonic SCs were cultured as reported previously (Jessen et al., 1994; Dong et al., 1999; Lobsiger et al., 2000) at a density of $250-500$ cells $/ 5 \mu$ l. In both cases, $\sim 25 \%$ of sorted cells attached. Short-term cultures were fixed after 3-6 hr. Survival assays were performed as described previously (Jessen et al., 1994; Dong et al., 1999; Lobsiger et al., 2000). Results are expressed as percentage of p $75^{+}$cells surviving after $24 \mathrm{hr}$. The number of input $\mathrm{p} 75^{+}$cells was determined by counting sister plates after $3 \mathrm{hr}$ in culture. Sorted cells from E12 embryos were induced to differentiate with human recombinant glial growth factor 2 (GGF2) (1 nM; from M. Marchionni, Cambridge NeuroScience, Cambridge, MA) plus $20 \mu \mathrm{M}$ forskolin for $4 \mathrm{~d}$.

The SC precursor cell line SpL201 (Lobsiger et al., 2001) was used at passage 17. RNA was isolated from cells cultured under normal growth conditions or after treatment with $20 \mu \mathrm{M}$ forskolin for $7 \mathrm{~d}$.

Immunohistochemistry. Immunohistochemistry on dissociated cells was performed as described (Lobsiger et al., 2000; Paratore et al., 2001). Antibodies were against O4 (mouse monoclonal, IgM, 1:10 dilution; from M. Schwab, Brain Research Institute, Zürich, Switzerland), p75ex (rabbit anti-mouse p75 extracellular domain, 1:500; Calbiochem, Lucerne, Switzerland), and Sox10 (mouse monoclonal, 1:3; from M. Wegner, University of Erlangen, Erlangen, Germany).

Microarray hybridization. Total RNA isolated with the RNeasy mini kit (Qiagen, Hilden, Germany) was amplified as described (Hoffmann et al., 2002), except that RNA was precipitated with ethanol after the Centricon ultrafiltration steps. Biotin-labeled, amplified cRNA $(8-15 \mu \mathrm{g})$ was fragmented and hybridized to Affymetrix mouse genome U74Av2 chips (catalog \#900343) according to the Affymetrix protocol (Affymetrix, Inc., 2000) on an Affymetrix GeneChip system (Lipshutz et al., 1999) at the Functional Genomics Center (Zürich).

Data analysis. CEL files obtained using MAS 5.0 (Affymetrix) were analyzed with the program dChip (Li and Wong, 2001a). After normalization (Li and Wong, 2001b), probe-sensitivity indices, characterizing the response of individual oligonucleotide probes across arrays, were used to calculate the "model-based expression index" using the perfect match/mismatch model (Li and Wong, 2001a) as a measure of the expression level of the cognate gene in a particular chip experiment. Comparisons between stages ( $\mathrm{Li}$ and Wong, 2001b) were performed with dChip using criteria described in Results. Hierarchical clustering was performed with genes called "present" on the majority of replicate arrays for at least one developmental stage. Genes with highly variable expression indices between replicate arrays were eliminated by requiring the median over developmental stages of the within-replica (SD/mean) to be $<0.5$. After pooling replicates, genes with sufficient variability across stages were chosen by requiring the (SD/mean) across all stages to be $>0.4$ (and $<5$ ). Clustering of the resulting 1435 genes was performed using the average linkage method (Eisen et al., 1998).

In situ hybridization. In situ hybridization was performed as described in Wilkinson (1992) using digoxigenin-labeled RNA probes for putative neuronal cell adhesion molecule (from S. Mansour, University of Utah, Salt Lake City, UT), reelin (from A. Goffinet, Université Louvain Medical School, Brussels), serpine 2 (BE534374), and myelin basic protein (MBP; from A. Gow, Cleveland Clinic Foundation).

\section{Results}

\section{Characterization of GFP-positive cells}

We tested a transgenic mouse line in which $P l p$ promoter elements drive expression of GFP (Spassky et al., 2001). Green fluorescence was apparent in the region of the somites in whole embryos at E9.5 (Fig. 1A) and at E10.5 (data not shown) in a 

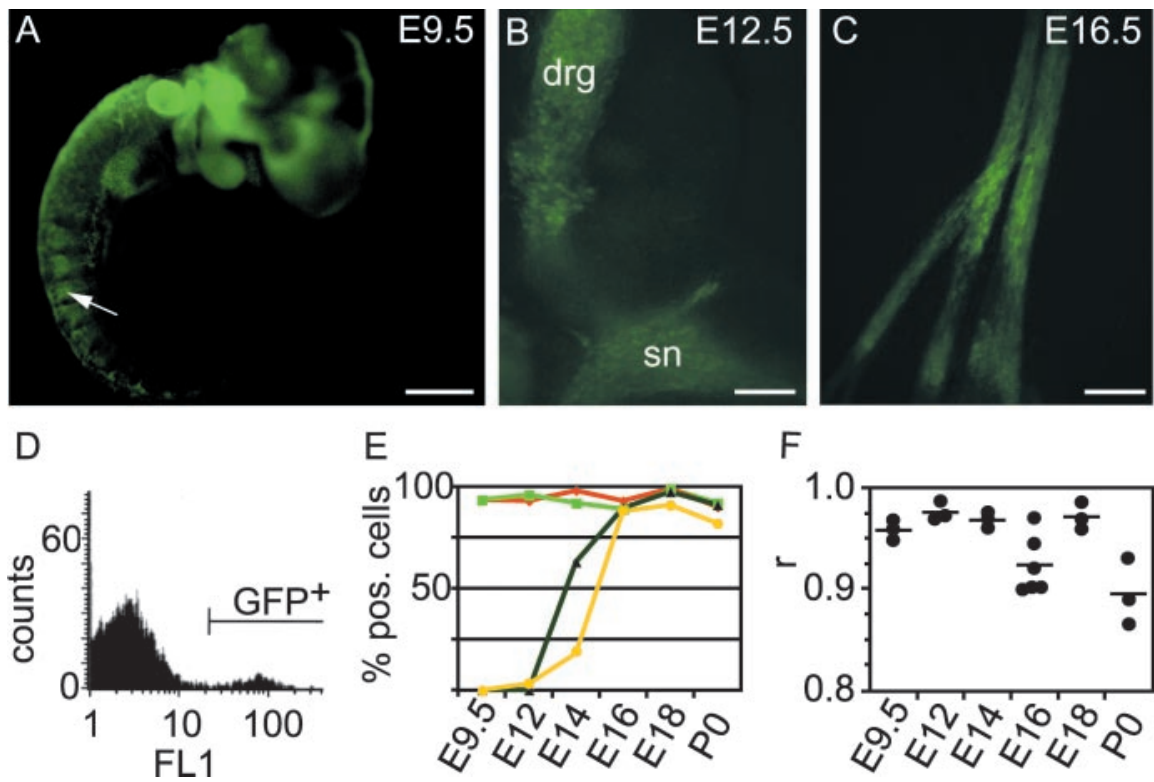

F

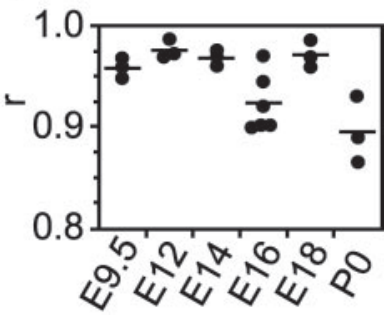

Figure 1. Expression of GFP in the developing PNS of Plp-GFP transgenic mice. A, At E9.5, GFP ${ }^{+}$cells are detected in the developing somites (arrow). B, At E12.5, GFP ${ }^{+}$cells are found in the DRG (drg) and around the sciatic nerve (sn) growing into the hindlimb. C, AtE16, GFP ${ }^{+}$cells are found within the sciatic nerve. Scale bars: $A, 0.5 \mathrm{~mm}, B, C, 200 \mu \mathrm{m}$. D, When analyzed by FACS, sciatic nerve cell suspensions obtained from transgenic mice at E16 show a distinct population of GFP ${ }^{+}$cells. E, Quantification of survival assays and immunohistochemical characterization of sorted cells. Expression of p75 (red diamonds) and Sox10 (green squares) is maintained by $\sim 90 \%$ of cells at each developmental stage, whereas expression of the 04 antigen (black triangles) begins around E14. At E14 a subpopulation of sorted cells acquires the ability to survive $24 \mathrm{hr}$ in culture without supplementation with growth factors, whereas at later stages almost all cells survive (yellow circles). $F$, Reproducibility of microarray hybridizations. Probes derived from RNA of sorted cells were hybridized to Affymetrix GeneChips, and expression indices were determined for all the genes. At each developmental stage, three or four replicate experiments were compared in all pairwise combinations by calculating Pearson's correlation coefficient for the expression indices. All genes on the chips were included in the comparisons. The filled circles show the correlation coefficients $(r)$, with three or six values for three or four replicas, respectively, and the horizontal lines indicate the means of these values.

pattern characteristic of migrating NCSCs. Developing sciatic nerves were positive by E12.5 and at later stages (Fig. $1 \mathrm{~B}, \mathrm{C}$ ). Thus, GFP was expressed at anatomical positions where NCSCs and cells of the SC lineage are expected.

Next, homozygous Plp-GFP males were mated with wild-type females, and transgenic embryos were collected at various stages. At E9.5 the trunk was isolated, whereas at later stages sciatic nerves were dissected. At all stages FACS analysis showed a distinct population of $\mathrm{GFP}^{+}$cells (Fig. $1 D$ ), comprising on average $20 \%$ of the input (supplemental Table 1, available at www.jneurosci.org). Such cells were not found with wild-type control embryos (data not shown). Aliquots of $\mathrm{GFP}^{+}$cells were seeded on coverslips, and the attached cells were tested further for characteristics typical of their respective stages in the glial lineage (two to four experiments per developmental stage; counting at least 250 cells per culture dish).

The great majority of sorted cells (85-99\%) were positive for the low-affinity neurotrophin receptor p75 and the transcription factor Sox10 at all stages examined (Fig. $1 E$, red diamonds and green squares, respectively), consistent with previous studies (Kuhlbrodt et al., 1998; Lobsiger et al., 2000; Paratore et al., 2001). The $\mathrm{O} 4$ antigen sulfatide is a differentiation marker absent from SC precursors but present in $~ 90 \%$ of early SCs isolated at E16 (Dong et al., 1999). We observed the same developmental course of antigen expression (Fig. $1 E$, black triangles).

SC precursors die within $24 \mathrm{hr}$ in culture if not provided with specific growth factors, whereas early SCs survive (Jessen et al., 1994; Dong et al., 1999; Lobsiger et al., 2000). As expected, sur- vival capacity of sorted cells was acquired between E12 and E16, with the E14 stage being intermediate (Fig. $1 E$, yellow circles). Furthermore, after culture for $4 \mathrm{~d}$ in the presence of forskolin and the growth and differentiation factor GGF2, the cells were $\mathrm{O} 4$ positive and exhibited the typical morphology of early SCs (data not shown).

We conclude that the GFP marker permits isolation of highly purified homogeneous populations of NCSCs and cells of the SC lineage.

\section{Target preparation and validation of the amplification procedure}

The limited amounts of RNA available from the sorted cells necessitated the use of an amplification procedure involving two rounds of in vitro transcription with $\mathrm{T} 7$ RNA polymerase (Van Gelder et al., 1990; Hoffmann et al., 2002). We compared this method with the standard one-round protocol using the SC precursor cell line SpL201, which can be induced by treatment with forskolin to differentiate into an early SC-like state (Lobsiger et al., 2001). Labeled cRNA was generated from forskolin-treated and control cells using either $5 \mu \mathrm{g}$ RNA and one round of in vitro transcription or $300 \mathrm{ng}$ RNA and two rounds. After hybridization to Affymetrix MGU74Av2 arrays, expression indices were computed using dChip software ( $\mathrm{Li}$ and Wong, 2001a). The results were similar for the two amplification methods ( $r=0.87-0.88)$, within the range of values reported for other two-round protocols [0.7-0.77 in Luzzi et al. (2003); 0.898 in Baugh et al. (2001); 0.9 in Affymetrix Inc. (2002)]. Large gene-specific differences in amplification efficiency were infrequent. Only 1.3\% (167 of 12488) of all genes repeatedly differed by $>1.5$-fold with targets prepared with one versus two rounds of in vitro transcription, using RNA from either forskolin-treated or control cells (fold changes are given as the $90 \%$ lower confidence bound of the change; see below). Similar changes in expression after forskolin treatment were seen with both methods, because 29 of 42 (69\%) genes called threefold or more changed between amplified samples were found to have changed by at least 1.5-fold between unamplified samples. Thus for most genes, the amplification system faithfully reproduces absolute expression levels and differences in expression levels between samples.

\section{Reproducibility of array hybridizations}

We performed three to four separate cell isolation and array hybridization experiments at each developmental stage. Pools of 6-25 embryos gave $\sim 7000-55,000$ sorted cells, and amplification of total RNA from these cells yielded 8-82 $\mu \mathrm{g}$ of biotinlabeled cRNA (supplemental Table 1, available at www.jneurosci.org) for hybridization.

Overall, 38-55\% of genes were called present for a set of 20 arrays (supplemental Table 1, available at www.jneurosci.org). We excluded a further array (P0_2) because only $24 \%$ of the genes were called present. Replica experiments displayed good 
A Peripheral myelin protein, $22 \mathrm{kDa}, 102395$ at

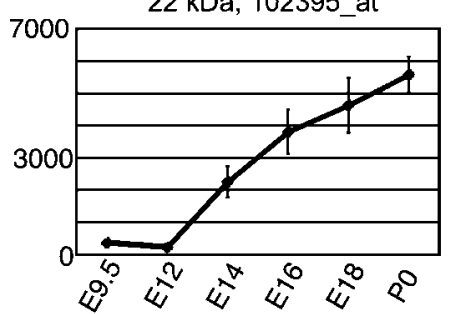

C Fatty acid binding protein 7, brain, 98967_at

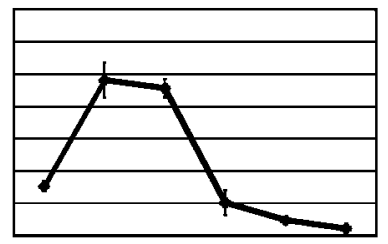

E Prion protein, 100606_at

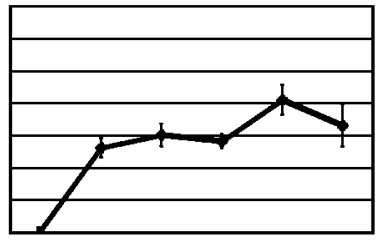

B Early growth response 2, 102661_at

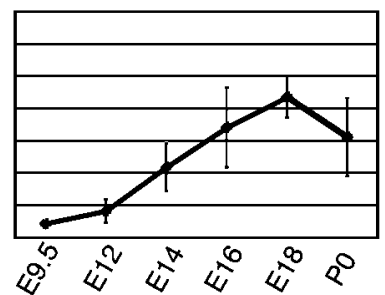

D Putative neuronal cell adhesion molecule, 94117 f_at

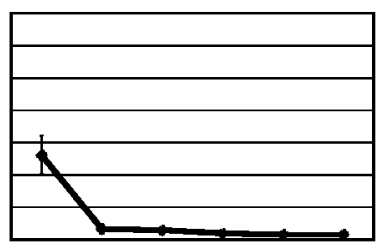

F

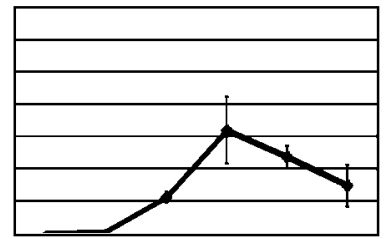

Figure 2. Expression profiles of genes in $\mathrm{SC}$ development. Profiles of three genes known to be involved in SC development are shown: peripheral myelin protein $22(A)$, early growth response $2(B)$, and fatty acid binding protein $7(C)$. Profiles of three genes not previously known to be expressed in the early $\mathrm{SC}$ lineage are shown: putative neuronal cell adhesion molecule (punc) ( $D$ ), prion protein $(E)$, and reelin ( $F$. Affymetrix probe-set identification numbers are above the graphs. Mean expression indices $\pm S D$ are shown.

reproducibility, with pairwise correlation coefficients ranging from 0.866 to 0.986 (Fig. $1 F$ ), except for one array (E12_3) that was discarded as an outlier $(r<0.8$; data not shown). Large variations between duplicates were rare. Further analysis was based on data obtained with 19 arrays (supplemental Table 1 shows list of arrays; primary data are available from Gene Expression Omnibus, http://www.ncbi.nlm.nih.gov/geo/, under accession numbers GSM15370 to GSM15388).

\section{Validation of expression data}

We sought to validate our expression data by comparison with earlier studies. In 32 of 35 cases (91\%), our data followed the previously described trends in expression (supplemental Table 2, available at www.jneurosci.org) (Fig. 2). These include genes encoding myelin structural proteins [peripheral myelin protein 22 (PMP22), myelin protein zero (MPZ), PLP and its splice variant DM-20], transcription factors involved in neural crest fate determination (N-myc, activating protein 2) or in myelin formation [Oct-6, early growth response 2 (EGR-2)], signaling molecules (endothelin, insulin-like growth factor 2), and basal lamina components and receptors (laminin, dystroglycan).

In situ hybridization and immunohistochemistry were used to assess with cellular resolution the expression of a number of genes not studied previously in the context of early Schwann cell development.

On the basis of the array analysis, putative neuronal cell adhesion molecule (punc) (Salbaum, 1998) is strongly expressed in NCSCs but subsequently downregulated during SC development (Fig. 2D). In situ hybridization revealed expression in emigrating

NCSCs at E9.5 (Fig. 3A) and weakly in E12 peripheral nerve (data not shown), in concordance with the results of Salbaum (1998).

Among the transcripts strongly upregulated during the transition from NCSCs at E9.5 to SC precursors at E12 (Fig. 2 E and data not shown) were those encoding the protease inhibitor serpine 2 (Mansuy et al., 1993), the transcription factor nuclear factor IB (NFI-B) (Chaudhry et al., 1997), the cellular prion protein (for review, see Behrens and Aguzzi, 2002), and astrotactin, a protein involved in neuronal migration (Zheng et al., 1996). Expression of serpine 2 and NFI-B was described previously in DRG and the surrounding mesenchyme at E12.5 (Mansuy et al., 1993; Chaudhry et al., 1997). We now found using in situ hybridization for serpine 2 and immunohistochemistry for NFI-B that both are also expressed in peripheral nerve at E12 (Fig. 3C,D and data not shown). Similarly, expression of astrotactin, previously not described in the peripheral nervous system, was detected in the neural tube, in DRG, and in peripheral nerves (data not shown). Immunohistochemistry was used to demonstrate the presence of cellular prion protein in presumptive Schwann cell precursor cells at E12.5 (data not shown).

Genes expressed at low or intermediate levels at E9.5 and E12, but strongly upregulated in the early SCs at E16 (Fig. $2 \mathrm{~F}$ and data not shown), included the soluble lectin galectin I (Horie and Kadoya, 2000), desmoyokin, a target of S100b in rat astrocytoma cells (Gentil et al., 2001), and the serine protease reelin, which regulates neuronal migration in the cortex (Rice and Curran, 2001). In situ hybridization confirmed expression of these genes in peripheral nerves at E16, but not at E12 (Fig. 3E and data not shown); the expression of reelin has been reported previously (Ikeda and Terashima, 1997).

We found that the cell cycle regulator p57, however, gave no specific in situ hybridization signal in developing peripheral nerves, although expression was detected in the surrounding mesenchyme (data not shown). Thus eight of nine (89\%) candidates were expressed in NCSCs and peripheral nerves at the predicted developmental stages.

\section{Global patterns of gene expression}

We screened for changes in expression between developmental stages using the program dChip (Li and Wong, 2001a). Comparisons between stages were performed applying conservative criteria, all of which take expression levels and variability at the single probe level into consideration (Li and Wong, 2001a,b) and were chosen to minimize the number of false positives. (1) Weighted average differences in expression indices were $>100$. This avoids very small changes near the noise level, at the expense of missing genes expressed only at low levels. (2) Expression was detected (called present) in at least $50 \%$ of arrays being compared. (3) A significance level of $p<0.05$ was attained in a modified unpaired $t$ test (Li and Wong, 2001b). (4) We used the $90 \%$ lower confidence bound of the fold change (LCB) rather than simply the fold change. Costigan et al. (2002) estimated a false discovery rate of 5\% among genes differentially expressed when they combined requirements of fold change $>1.5$ and $p<0.05$ for individual genes.

From these considerations and the results of the previous section, we conclude that our analysis provides a firm basis for selection of candidate genes.

We subsequently grouped the differentially expressed genes into functional categories (supplemental Table 3, available at www.jneurosci.org). Changes were most frequent during the transition from migrating NCSCs at E9.5 to SC precursors at E12 (Fig. 4A). Strikingly more genes were upregulated rather than 

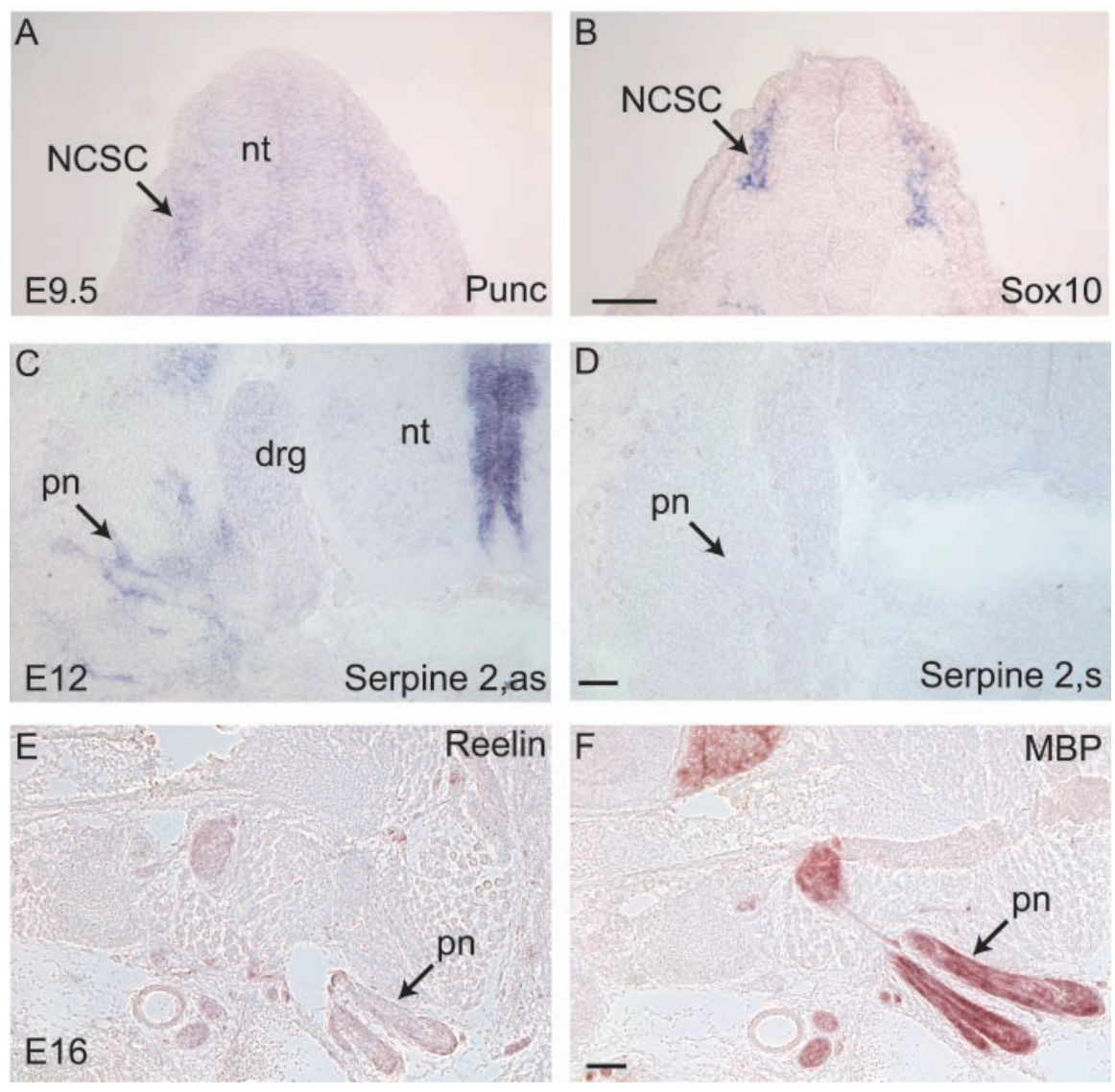

Figure 3. Expression of candidate genes assessed by in situ hybridization. $A$, Expression of punc $m R N A$ is detected at E9.5 in migrating NCSCs of the trunk (arrow), which were identified by expression of Sox10 on nearly adjacent sections (B). C, Serpine 2 is found in E12 peripheral nerves ( $\mathrm{pn}$, arrow); hybridization with the serpine 2 sense control probe is shown in D. E, Reelin is expressed in E16 peripheral nerves. Location of peripheral nerves was determined by hybridization of a probe for MBP mRNA to semi-adjacent sections (F). Scale bar, $100 \mu \mathrm{m}$. NCSC, Neural crest stem cells; nt, neural tube; drg, DRG; pn, peripheral nerve.

downregulated. This difference held across all classes of genes considered, including genes encoding regulatory molecules such as transcription factors, regulators of the cell cycle, growth and differentiation factors, and kinases and phosphatases (Fig. 4B).

Changes in some groups were concentrated between particular stages (Fig. 4C). Upregulation of various extracellular matrix (ECM) components (tenascin C, laminin chains, and some collagen isoforms) and receptors (integrin $\alpha 4$ ) previously reported to be involved in NCSC survival and migration (Haack and Hynes, 2001; Previtali et al., 2001) started as early as E9.5-E12. Fibronectin and integrin $\alpha 4$ were downregulated from E12 to E14. Molecules contributing to the basal lamina of axon bundles (laminin chains, various collagen isoforms, and proteoglycans such as decorin) and their receptors (dystroglycan 1, integrin $\beta 4$ ) were strongly and continuously upregulated from E12 to E16. A peak of expression of ECM-related transcripts was seen at E16 or E18, a period when the basal lamina is formed around the nerve fibers (Previtali et al., 2001). Increases in transcripts encoding myelin components (PMP22, MBP, myelin and lymphocyte protein) were seen as early as E12 and continued to increase until E16 or later, consistent with previous findings (Blanchard et al., 1996; Hagedorn et al., 1999). Molecules involved in cytoskeletal rearrangements were mainly found differentially expressed from E9.5 to E12 and E12 to E14, during the transition of migratory NCSCs to the still highly migratory SC precursor cells (Jessen et al., 1994). Expression of cell cycle regulators remained relatively con- stant from E12 to E18, whereas negative cell cycle regulators (p107, Brca 1) were downregulated between E18 and P0, a developmental phase in which early SCs proliferate extensively (Stewart et al., 1993).

To follow patterns of expression over multiple stages, 1435 selected genes (see Materials and Methods) were subjected to hierarchical clustering. We readily identified five major clusters (Fig. 4D) (supplemental Table 4, available at www.jneurosci.org), with expression patterns suggestive of roles in specific developmental stages (see Discussion). Among genes present in the clusters are many expressed at low levels or displaying smaller fluctuations over time. We therefore sought to select genes with the most substantial variation in expression by combining the clustering and stage-to-stage comparisons (supplemental Table 5, available at www. jneurosci.org). For example, the 512 genes in cluster 1 are expressed most strongly in NCSCs and at reduced levels thereafter. Of these, 205 overlap with those found to decrease between E9.5 and E12 in the stageto-stage comparison, the rest representing genes with low expression or with LCB $<1.5$. Similarly, a subset of cluster 2 was chosen by requiring that expression decreased significantly from E12 to E14. For cluster 3 we added the condition that expression increased between E9.5 and either E12 or E14, but then decreased by E16. Clusters 4 and 5 were combined and subjected to the further requirement that expression increased after E12. These cluster subsets thus contain genes with substantial peaks in expression at particular developmental stages.

\section{Discussion}

A mouse line expressing GFP under the control of the Plp promoter was used for rapid isolation of migratory neural crest stem cells and cells of the developing Schwann cell lineage. The fluorescent reporter offered multiple advantages. (1) We had access to a pure population of neural crest stem cells, which, being dispersed throughout the somites, are not directly dissectable ex vivo. (2) Non-glial cells were effectively eliminated from sciatic nerve preparations at later stages (Fig. 1E). (3) We could undertake cell sorting immediately after dissection and dissociation of the tissue, without the need for prolonged cell culture or incubation with antibodies.

To increase the significance of our expression data we performed three to four replica array hybridizations using RNA preparations obtained from separate batches of embryos. Except for two outliers, these biological replicates displayed good reproducibility, with pairwise correlation coefficients ranging from 0.866 to 0.986 (Fig. $1 F$ ). The number of genes with expression indices differing substantially between replicas was low, $0.2-1 \%$ of the total for threefold or greater differences at stages up to E18, and $2.2 \%$ at $\mathrm{PO}$ (data not shown). The greater variation seen at $\mathrm{P} 0$ may reflect true biological variability, possibly in exact age when 
mice are killed, because some of the litters collected on day P0 may have been born in the evening and others toward morning.

Literature comparisons and either immunohistochemistry or in situ hybridization were used to validate the expression data, with the in situ methods providing controls for both the microarray analysis and the cell purification method. Most genes for which we could find at least semiquantitative data in the literature were also identified in our screen. Furthermore, we could validate the expression of eight of nine genes by in situ hybridization or immunohistochemistry. Three of the transcripts that we detected by in situ hybridization were predicted to be expressed at rather low levels, namely punc (detected at E9.5 and E12; mean expression index of 325 at E12), nuclear factor IB (mean expression index 302 at E12), and astrotactin (mean expression index of 512 at E12). This suggests that candidate genes can be chosen even on the basis of relatively low expression indices, confirming conclusions drawn by others using "spike" experiments (Lockhart et al., 1996; Baugh et al., 2001). The possibility remains, however, that gene-specific differences in amplification efficiency could affect the choice of candidate genes expressed at very low levels, either because despite amplification the signal remains below the noise level or because differences in expression between developmental stages fall below the threshold difference that we used. With in situ hybridization, only one probe of the set that we tested gave a positive signal in surrounding tissue but not in nerve. We conclude that our screening procedure can reliably identify genes expressed in the $\mathrm{SC}$ lineage. Others have validated GeneChip results by literature screens, in situ hybridization, Northern blotting, and quantitative slot blots, and found, similar to our results, $60-83 \%$ concordance of detection (Zirlinger et al., 2001; Costigan et al., 2002; Hoffmann et al., 2002).

Stage-to-stage comparisons and hierarchical clustering revealed that changes in expression were most frequent between E9.5 and E12. Substantial increases in expression were concentrated here (Fig. 4A), but small decreases were also common (Fig. 4D). A similar pattern was seen by Luo et al. (2002), who found stem cells to express a wide variety of transcripts at low levels, whereas differentiated cells expressed a smaller number of transcripts but at higher levels. From E12 to E14 the number of genes changing by $>1.5$-fold remained in the hundreds, whereas it decreased below 100 after E14. Changes were much less frequent from E16 on, when the nerve is populated by a more homogenous population of immature SCs (Figs. 1E, 4A) (Dong et al., 1999).
A Number of genes differentially expressed between developmental stages

\begin{tabular}{|l|r|r|r|}
\hline LCB > 1.5 & total & up & down \\
\hline E9.5 vs E12 & 915 & 655 & 260 \\
\hline E12 vs E14 & 201 & 102 & 99 \\
\hline E14 vs E16 & 169 & 60 & 109 \\
\hline E16 vs E18 & 35 & 17 & 18 \\
\hline E18 vs P0 & 49 & 12 & 37 \\
\hline
\end{tabular}

B Classes of genes differentially expressed between E9.5 and E12

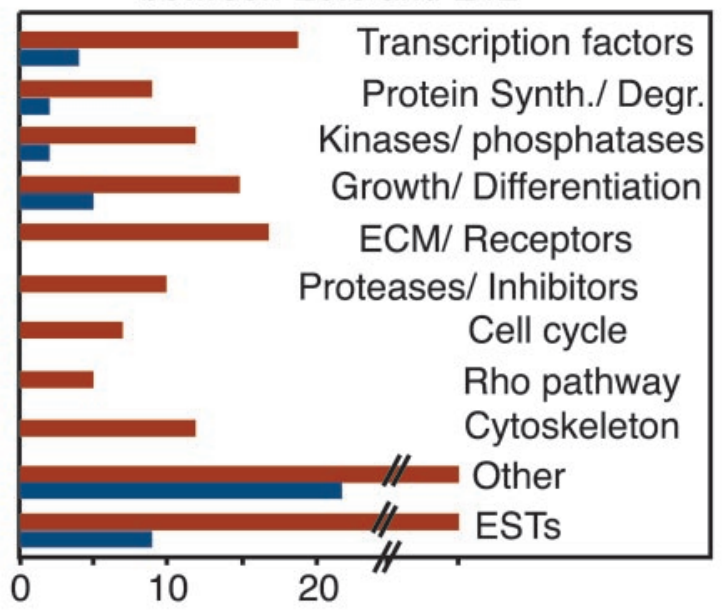

C Categories of genes differentially expressed between developmental stages

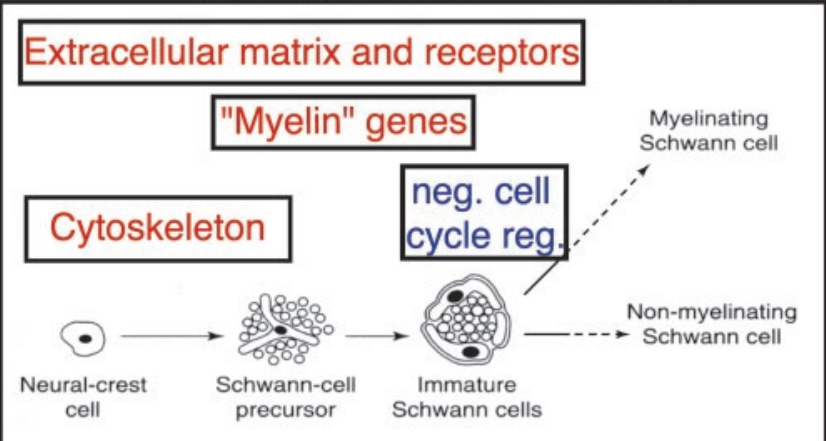

E9.5 E12 E14 E16 E18 P0

Figure 4. $A$, Number of genes differentially expressed with an $L C B$ of $\geq 1.5$-fold between developmental stages (additional criteria: change in expression index $\geq 100 ; p \leq 0.05$ by modified $t$ test; genes called present on at least $50 \%$ of arrays used for the comparison). $B$, Categories of genes differentially expressed between $E 9.5$ and $E 12$ with $L C B \geq 3 \times$, or $\geq 2 \times$ if the mean expression index was $>1000$ for at least one stage. $C$, Genes differentially expressed between developmental stages were grouped into functional categories. The most prominent groups regulated through SC development are indicated in blue for genes in these groups mainly downregulated or in red if mainly upregulated. Diagram of developmental stages adapted from Jessen and Mirsky (1999). D, Hierarchical clustering of expression patterns of genes called present for at least one developmental stage. Columns represent expression levels at the various developmental stage; rows correspond to individual genes. Expression levels are relative to the mean across stages, normalized to have a mean of 0 and an SD of 1 . Expression levels are color coded relative to the mean: blue for values less than the mean and red for values larger than the mean. The scale indicates SDs above or below the mean. Clusters discussed in Results are numbered 1-5.

Both the clusters and the stage-to-stage comparison were used to select novel candidate genes (supplemental Tables 3-5, available at www.jneurosci.org). At present, it is only partially clear how and when lineage specification of neural crest-derived cells into the SC lineage occurs. The TGF $\beta$ family (Shah et al., 1996; Hagedorn et al., 1999, 2000) and components of the Wnt signaling pathway (Hari et al., 2002) play a role in early neural crest 
lineage specification. Notch signaling (Morrison et al., 2000) and neuregulins (Shah et al., 1994; Leimeroth et al., 2002) are active in early glial fate determination and differentiation in the PNS. Clusters 2 and 3, comprising genes with peak expression in NCSCs and SC precursors, include components of the Wnt (dickkopf 2, secreted frizzled-related sequence protein, Wnt1responsive Cdc42 homolog, T-cell factor 4 ) and TGF $\beta$ (inhibitor of DNA binding 1 and 3, TGF $\beta 1$-induced transcript 4, disabled homolog 2) pathways. These genes might be involved in the suppression of differentiation from NCSCs into neurons. Additionally we found a transducer of the Notch signaling cascade (transducin-like enhancer of split), which may regulate target genes necessary for glial fate specification. Many novel receptor protein kinases and phosphatases are found in clusters $2 / 3$ or are differentially expressed between E9.5 and E12 (such as protein tyrosine phosphatase types D, E, and $\mathrm{M}$, and receptor tyrosine kinase-like orphan receptor), offering a number of additional candidates to be tested for a role in response to extracellular cues in lineage specification (supplemental Tables 3-5, available at www.jneurosci.org).

Current understanding of the cell-intrinsic factors integrating these extracellular cues is limited. Sox 10 is one transcription factor known to positively influence glial fate determination from NCSCs (Britsch et al., 2001; Paratore et al., 2001). In stage-tostage comparisons and in clusters 2 and 3 we find number of transcription factors highly expressed at E12 and downregulated by E16, which could be involved in glial fate specification or early differentiation processes. These include members of the homeobox (distal-less homeobox-1 and 2), ets-domain (E74-like factor 1), Krüppel-like (Klf-3), basic helix-loop-helix (T-cell factor 4, liver-specific bHLH-Zip transcription factor), and others [the Egr-1/2 binding protein Nab1, the cAMP response element modulator (Crem), the nuclear receptor interacting protein 1 (Nrip1), and nuclear receptor coactivator 6 (Ncoa6) ]. Dlx-1 expression has been detected previously at E11.5 and E12.5 in parts of the nerve trunks from the brachial plexus and in the roots of the sciatic nerve, which are populated by Schwann cell precursor cells at this age (Stewart et al., 1996). Because cAMP levels are known to be important in regulating SC differentiation (Jessen and Mirsky, 1998), Crem might play a role in Schwann cell response to cAMP already at this early stage of development (Stewart et al., 1996).

Clusters 4 and 5 both contain genes expressed at low levels in early stages of SC development and upregulated after E12. Among these genes we find, as expected and already seen in stageto-stage comparisons, a large number of transcripts encoding ECM molecules (laminin $\alpha 2$ and $\beta 1$; various collagens) and their receptors (dystroglycan 1 , integrin $\beta 4$ ). Additionally, soluble or cell-surface proteoglycans (syndecan 4, glypican 4, decorin, SPARC-like 1) acting as ligands for ECM molecules and growth factors, such as fibroblast growth factor and vascular endothelial growth factor (Bernfield et al., 1999; Brekken and Sage, 2001) were upregulated between E12 and E14. These molecules could be further investigated as modulators of growth factor-receptor interactions during early Schwann cell development. Genes encoding myelin components (PLP, PMP22, MBP) are found highly expressed from E14 on until P0. The transcription factor Egr-2, known to be involved in myelination, is in cluster 5 along with many other transcription factors possibly involved in the transcriptional network regulating myelination or extracellular matrix synthesis [Krüppel-like factors 7 and 9; zinc finger proteins 36 and 103; a B-class basic helix-loop-helix protein (Stra13)]. Genes causing peripheral neuropathies when mutated [lamin A, N-myc downstream-regulated gene 1, periaxin, MPZ, PMP22; (Suter and Scherer, 2003)] are also found in cluster 5. Genes encoding cell cycle and cytoskeletal molecules are present in all clusters, reflecting the fact that we have profiled a migratory and proliferating cell population.

We compared cluster 1 with genes previously found specifically enriched in mouse CNS neural stem cells cultured as neurospheres (Ramalho-Santos et al., 2002). Of the genes in cluster 1, $63 \%$ ( 320 of 512) were among the 2458 genes found enriched in neurospheres, and $36 \%$ were common to a group of 230 found common to neurospheres, embryonal stem cells, and hematopoietic stem cells (Ramalho-Santos et al., 2002) (supplemental Table 6 , available at www.jneurosci.org). The overlap is highly significant in both cases $\left(p<10^{-12}\right.$; hypergeometric distribution). Among the genes expressed by the different stem cell populations, we found genes involved in the regulation of cell cycle progression (Xrcc5), chromatin remodeling factors (enhancer trap locus 1), and the general transcription-translation process (bystin-like, RNA cyclase homolog). A large number of genes found in cluster 1 were related to cell metabolism, DNA repair, chromatin remodeling and replication, or to general transcription-/translation processes. Similar patterns have been reported for neural stem cells from neonatal mouse neocortex cultured as neurospheres when compared with differentiating cells (Karsten et al., 2003). Expression of genes in cluster 2 is relatively high at E9.5 and E12 and declines later. Some of these genes may still be involved in maintaining a stem cell or progenitor state, because multipotent stem cells are also present at later stages in embryonic sciatic nerve (Morrison et al., 1999).

\section{Conclusion}

Transgenic mice expressing GFP in a tissue-specific manner allowed us to isolate homogeneous cell populations of NCSCs and developing SCs, which are not readily accessible by microdissection. Reproducible array hybridizations could be performed using a two-round amplification procedure, starting with small amounts of RNA. We identified many genes already known to be involved in fate determination, differentiation, and maturation of glial cells, thus validating the cell isolation and screening procedure. Stage-to-stage comparisons and hierarchical clustering identified a number of candidates involved in the regulation of early stem cells and SC development. We confirmed by in situ hybridization the differential expression of some of these candidates that belong to gene families not previously thought to function in SC development. Further functional tests will undoubtedly reveal new regulatory factors involved in PNS development.

\section{References}

Affymetrix Inc. (2000) Expression analysis: technical manual.

Affymetrix Inc. (2002) GeneChip eukaryotic small sample target labeling assay version II, Technical Note 701265, Rev 3.

Anderson DJ (1997) Cellular and molecular biology of neural crest cell lineage determination. Trends Genet 13:276-280.

Anderson DJ (2000) Genes, lineages and the neural crest: a speculative review. Philos Trans R Soc Lond B Biol Sci 355:953-964.

Baugh LR, Hill AA, Brown EL, Hunter CP (2001) Quantitative analysis of mRNA amplification by in vitro transcription. Nucleic Acids Res 29:E29-E38.

Behrens A, Aguzzi A (2002) Small is not beautiful: antagonizing functions for the prion protein $\operatorname{PrP}(\mathrm{C})$ and its homologue Dpl. Trends Neurosci 25:150-154.

Bernfield M, Gotte M, Park PW, Reizes O, Fitzgerald ML, Lincecum J, Zako M (1999) Functions of cell surface heparan sulfate proteoglycans. Annu Rev Biochem 68:729-777.

Blanchard AD, Sinanan A, Parmantier E, Zwart R, Broos L, Meijer D, Meier C, 
Jessen KR, Mirsky R (1996) Oct-6 (SCIP/Tst-1) is expressed in Schwann cell precursors, embryonic Schwann cells, and postnatal myelinating Schwann cells: comparison with Oct-1, Krox-20, and Pax-3. J Neurosci Res 46:630-640.

Brekken RA, Sage EH (2001) SPARC, a matricellular protein: at the crossroads of cell-matrix communication. Matrix Biol 19:816-827.

Britsch S, Goerich DE, Riethmacher D, Peirano RI, Rossner M, Nave KA, Birchmeier C, Wegner M (2001) The transcription factor Sox10 is a key regulator of peripheral glial development. Genes Dev 15:66-78.

Chaudhry AZ, Lyons GE, Gronostajski RM (1997) Expression patterns of the four nuclear factor I genes during mouse embryogenesis indicate a potential role in development. Dev Dyn 208:313-325.

Costigan M, Befort K, Karchewski L, Griffin RS, D’Urso D, Allchorne A, Sitarski J, Mannion JW, Pratt RE, Woolf CJ (2002) Replicate highdensity rat genome oligonucleotide microarrays reveal hundreds of regulated genes in the dorsal root ganglion after peripheral nerve injury. BMC Neurosci 3:16-33.

Davies AM (1998) Developmental changes in the neurotrophic factor survival requirements of peripheral nervous system neurons. Prog Brain Res 117:47-56.

Dong Z, Brennan A, Liu N, Yarden Y, Lefkowitz G, Mirsky R, Jessen KR (1995) Neu differentiation factor is a neuron-glia signal and regulates survival, proliferation, and maturation of rat Schwann cell precursors. Neuron 15:585-596.

Dong Z, Sinanan A, Parkinson D, Parmantier E, Mirsky R, Jessen KR (1999) Schwann cell development in embryonic mouse nerves. J Neurosci Res 56:334-348.

Eisen MB, Spellman PT, Brown PO, Botstein D (1998) Cluster analysis and display of genome-wide expression patterns. Proc Natl Acad Sci USA 95:14863-14868.

Fuss B, Mallon B, Phan T, Ohlemeyer C, Kirchhoff F, Nishiyama A, Macklin WB (2000) Purification and analysis of in vivo-differentiated oligodendrocytes expressing the green fluorescent protein. Dev Biol 218:259-274.

Gammill LS, Bronner-Fraser M (2002) Genomic analysis of neural crest induction. Development 129:5731-5741.

Garratt AN, Voiculescu O, Topilko P, Charnay P, Birchmeier C (2000) A dual role of erbB2 in myelination and in expansion of the Schwann cell precursor pool. J Cell Biol 148:1035-1046.

Gentil BJ, Delphin C, Mbele GO, Deloulme JC, Ferro M, Garin J, Baudier J (2001) The giant protein AHNAK is a specific target for the calcium- and zinc-binding S100B protein: potential implications for $\mathrm{Ca}^{2+}$ homeostasis regulation by S100B. J Biol Chem 276:23253-23261.

Griffiths I, Klugmann M, Anderson T, Thomson C, Vouyiouklis D, Nave KA (1998) Current concepts of PLP and its role in the nervous system. Microsc Res Tech 41:344-358.

Haack H, Hynes RO (2001) Integrin receptors are required for cell survival and proliferation during development of the peripheral glial lineage. Dev Biol 233:38-55.

Hagedorn L, Suter U, Sommer L (1999) P0 and PMP22 mark a multipotent neural crest-derived cell type that displays community effects in response to TGF-beta family factors. Development 126:3781-3794.

Hagedorn L, Floris J, Suter U, Sommer L (2000) Autonomic neurogenesis and apoptosis are alternative fates of progenitor cell communities induced by TGFbeta. Dev Biol 228:57-72.

Hari L, Brault V, Kleber M, Lee HY, Ille F, Leimeroth R, Paratore C, Suter U, Kemler R, Sommer L (2002) Lineage-specific requirements of betacatenin in neural crest development. J Cell Biol 159:867-880.

Hoffmann R, Seidl T, Neeb M, Rolink A, Melchers F (2002) Changes in gene expression profiles in developing $B$ cells of murine bone marrow. Genome Res 12:98-111.

Horie H, Kadoya T (2000) Identification of oxidized galectin-1 as an initial repair regulatory factor after axotomy in peripheral nerves. Neurosci Res 38:131-137.

Ikeda Y, Terashima T (1997) Expression of reelin, the gene responsible for the reeler mutation, in embryonic development and adulthood in the mouse. Dev Dyn 210:157-172.

Jessen KR, Mirsky R (1998) Origin and early development of Schwann cells. Microsc Res Tech 41:393-402.

Jessen KR, Mirsky R (1999) Schwann cells and their precursors emerge as major regulators of nerve development. Trends Neurosci 22:402-410.

Jessen KR, Mirsky R (2002) Signals that determine Schwann cell identity. J Anat 200:367-376.
Jessen KR, Brennan A, Morgan L, Mirsky R, Kent A, Hashimoto Y, Gavrilovic J (1994) The Schwann cell precursor and its fate: a study of cell death and differentiation during gliogenesis in rat embryonic nerves. Neuron 12:509-527.

Karsten SL, Kudo LC, Jackson R, Sabatti C, Kornblum HI, Geschwind DH (2003) Global analysis of gene expression in neural progenitors reveals specific cell-cycle, signaling, and metabolic networks. Dev Biol 261:165-182.

Kuhlbrodt K, Herbarth B, Sock E, Hermans-Borgmeyer I, Wegner M (1998) Sox10, a novel transcriptional modulator in glial cells. J Neurosci 18:237-250.

Le Douarin N, Kalcheim C (1999) The neural crest, Ed 2. Cambridge, MA: Cambridge UP.

Leimeroth R, Lobsiger C, Lussi A, Taylor V, Suter U, Sommer L (2002) Membrane-bound neuregulin1 type III actively promotes Schwann cell differentiation of multipotent progenitor cells. Dev Biol 246:245-258.

Li C, Wong WH (2001a) Model-based analysis of oligonucleotide arrays: expression index computation and outlier detection. Proc Natl Acad Sci USA 98:31-36.

Li C, Wong WH (2001b) Model-based analysis of oligonucleotide arrays: model validation, design issues and standard error application. Genome Biol 2:0032.1-0032.11.

Lipshutz RJ, Fodor SP, Gingeras TR, Lockhart DJ (1999) High density synthetic oligonucleotide arrays. Nat Genet 21:20-24.

Lobsiger CS, Schweitzer B, Taylor V, Suter U (2000) Platelet-derived growth factor-BB supports the survival of cultured rat Schwann cell precursors in synergy with neurotrophin-3. Glia 30:290-300.

Lobsiger CS, Smith PM, Buchstaller J, Schweitzer B, Franklin RJ, Suter U, Taylor V (2001) SpL201: a conditionally immortalized Schwann cell precursor line that generates myelin. Glia 36:31-47.

Lobsiger CS, Taylor V, Suter U (2002) The early life of a Schwann cell. Biol Chem 383:245-253.

Lockhart DJ, Dong H, Byrne MC, Follettie MT, Gallo MV, Chee MS, Mittmann M, Wang C, Kobayashi M, Horton H, Brown EL (1996) Expression monitoring by hybridization to high-density oligonucleotide arrays. Nat Biotechnol 14:1675-1680.

Luo Y, Cai J, Liu Y, Xue H, Chrest FJ, Wersto RP, Rao M (2002) Microarray analysis of selected genes in neural stem and progenitor cells. J Neurochem 83:1481-1497.

Luzzi V, Mahadevappa M, Raja R, Warrington JA, Watson MA (2003) Accurate and reproducible gene expression profiles from laser capture microdissection, transcript amplification, and high density oligonucleotide microarray analysis. J Mol Diagn 5:9-14.

Mansuy IM, van der Putten H, Schmid P, Meins M, Botteri FM, Monard D (1993) Variable and multiple expression of protease Nexin-1 during mouse organogenesis and nervous system development. Development 119:1119-1134.

Meyer D, Birchmeier C (1995) Multiple essential functions of neuregulin in development. Nature 378:386-390.

Morrison SJ (2001) Neuronal differentiation: proneural genes inhibit gliogenesis. Curr Biol 11:R349-351.

Morrison SJ, White PM, Zock C, Anderson DJ (1999) Prospective identification, isolation by flow cytometry, and in vivo self-renewal of multipotent mammalian neural crest stem cells. Cell 96:737-749.

Morrison SJ, Perez SE, Qiao Z, Verdi JM, Hicks C, Weinmaster G, Anderson DJ (2000) Transient Notch activation initiates an irreversible switch from neurogenesis to gliogenesis by neural crest stem cells. Cell 101:499-510.

Nagarajan R, Le N, Mahoney H, Araki T, Milbrandt J (2002) Deciphering peripheral nerve myelination by using Schwann cell expression profiling. Proc Natl Acad Sci USA 99:8998-9003.

Paratore C, Goerich DE, Suter U, Wegner M, Sommer L (2001) Survival and glial fate acquisition of neural crest cells are regulated by an interplay between the transcription factor Sox 10 and extrinsic combinatorial signaling. Development 128:3949-3961.

Parkinson DB, Dong Z, Bunting H, Whitfield J, Meier C, Marie H, Mirsky R, Jessen KR (2001) Transforming growth factor $\beta$ (TGF $\beta$ ) mediates Schwann cell death in vitro and in vivo: examination of c-Jun activation, interactions with survival signals, and the relationship of TGF $\beta$-mediated death to Schwann cell differentiation. J Neurosci 21:8572-8585.

Parmantier E, Lynn B, Lawson D, Turmaine M, Namini SS, Chakrabarti L, McMahon AP, Jessen KR, Mirsky R (1999) Schwann cell-derived Desert 
hedgehog controls the development of peripheral nerve sheaths. Neuron 23:713-724.

Previtali SC, Feltri ML, Archelos JJ, Quattrini A, Wrabetz L, Hartung H (2001) Role of integrins in the peripheral nervous system. Prog Neurobiol 64:35-49.

Ramalho-Santos M, Yoon S, Matsuzaki Y, Mulligan RC, Melton DA (2002) "Stemness": transcriptional profiling of embryonic and adult stem cells. Science 298:597-600.

Rice DS, Curran T (2001) Role of the reelin signaling pathway in central nervous system development. Annu Rev Neurosci 24:1005-1039.

Riethmacher D, Sonnenberg-Riethmacher E, Brinkmann V, Yamaai T, Lewin GR, Birchmeier C (1997) Severe neuropathies in mice with targeted mutations in the ErbB3 receptor. Nature 389:725-730.

Salbaum JM (1998) Punc, a novel mouse gene of the immunoglobulin superfamily, is expressed predominantly in the developing nervous system. Mech Dev 71:201-204.

Scherer SS, Arroyo EJ (2002) Recent progress on the molecular organization of myelinated axons. J Peripher Nerv Syst 7:1-12.

Shah NM, Marchionni MA, Isaacs I, Stroobant P, Anderson DJ (1994) Glial growth factor restricts mammalian neural crest stem cells to a glial fate. Cell 77:349-360.

Shah NM, Groves AK, Anderson DJ (1996) Alternative neural crest cell fates are instructively promoted by TGFbeta superfamily members. Cell 85 : 331-343.

Smith L, Greenfield A (2003) DNA microarrays and development. Hum Mol Genet 12:R1-R8.

Sommer L (2001) Context-dependent regulation of fate decisions in multipotent progenitor cells of the peripheral nervous system. Cell Tissue Res 305:211-216.

Spassky N, Olivier C, Cobos I, LeBras B, Goujet-Zalc C, Martinez S, Zalc B, Thomas JL (2001) The early steps of oligodendrogenesis: insights from the study of the plp lineage in the brain of chicks and rodents. Dev Neurosci 23:318-326.

Spiegel I, Peles E (2002) Cellular junctions of myelinated nerves. Mol Membr Biol 19:95-101.
Stemple DL, Anderson DJ (1992) Isolation of a stem cell for neurons and glia from the mammalian neural crest. Cell 71:973-985.

Stevens B, Fields RD (2000) Response of Schwann cells to action potentials in development. Science 287:2267-2271.

Stewart HJ, Morgan L, Jessen KR, Mirsky R (1993) Changes in DNA synthesis rate in the Schwann cell lineage in vivo are correlated with the precursor-Schwann cell transition and myelination. Eur J Neurosci 5:1136-1144.

Stewart HJ, Mirsky R, Jessen KR (1996) The Schwann cell lineage: embryonic and early postnatal development. In: Glial cell development (Jessen KR, Richardson WD, eds), pp 1-30. Oxford: BIOS.

Suter U, Scherer SS (2003) Disease mechanisms in inherited neuropathies. Nat Rev Neurosci 4:714-726.

Terenghi G (1999) Peripheral nerve regeneration and neurotrophic factors. J Anat 194:1-14.

Van Gelder RN, von Zastrow ME, Yool A, Dement WC, Barchas JD, Eberwine JH (1990) Amplified RNA synthesized from limited quantities of heterogeneous cDNA. Proc Natl Acad Sci USA 87:1663-1667.

Verheijen MH, Chrast R, Burrola P, Lemke G (2003) Local regulation of fat metabolism in peripheral nerves. Genes Dev 17:2450-2464.

Wakamatsu Y, Maynard TM, Weston JA (2000) Fate determination of neural crest cells by NOTCH-mediated lateral inhibition and asymmetrical cell division during gangliogenesis. Development 127:2811-2821.

Wilkinson DG (1992) Whole-mount in situ hybridization of vertebrate embryos. In: In situ hybridization: a practical approach (Wilkinson DG, ed), pp 75-83. Oxford: IRL.

Woldeyesus MT, Britsch S, Riethmacher D, Xu L, Sonnenberg-Riethmacher E, Abou-Rebyeh F, Harvey R, Caroni P, Birchmeier C (1999) Peripheral nervous system defects in erbB2 mutants following genetic rescue of heart development. Genes Dev 13:2538-2548.

Zheng C, Heintz N, Hatten ME (1996) CNS gene encoding astrotactin, which supports neuronal migration along glial fibers. Science 272:417-419.

Zirlinger M, Kreiman G, Anderson DJ (2001) Amygdala-enriched genes identified by microarray technology are restricted to specific amygdaloid subnuclei. Proc Natl Acad Sci USA 98:5270-5275. 